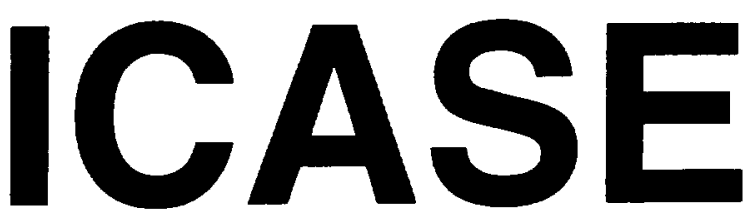

\title{
CONSISTENT INITIAL CONDITIONS FOR THE DNS OF COMPRESSIBLE TURBULENCE
}

\section{J. R. Ristorcelli \\ G. A. Blaisdell}

NASA Contract No. NAS1-19480

July 1996

Institute for Computer Applications in Science and Engineering

NASA Langley Research Center

Hampton, VA 23681-0001

Operated by Universities Space Research Association

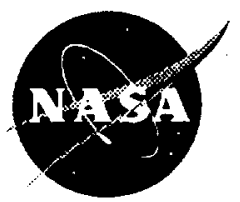

National Aeronautics and Space Administration

Langley Research Center Hampton, Virginia 23681-0001 



\title{
Consistent initial conditions for the DNS of compressible turbulence
}

\author{
J. R. Ristorcelli ${ }^{1}$ \\ Institute for Computer Applications in Science and Engineering \\ NASA Langley Research Center, Hampton, VA 23681-0001 \\ G. A. Blaisdell \\ School of Aeronautics and Astronautics \\ Purdue University, West Lafayette, IN 47907-1282
}

\begin{abstract}
Relationships between diverse thermodynamic quantities appropriate to weakly compressible turbulence are derived. It is shown that for turbulence of a finite turbulent Mach number there is a finite effect of compressibility. A methodology for generating initial conditions for the fluctuating pressure, density and dilatational velocity is given which is consistent with finite Mach number effects. Use of these initial conditions gives rise to a smooth development of the flow, in contrast to cases in which these fields are specified arbitrarily or set to zero. Comparisons of the effect of different types of initial conditions are made using direct numerical simulation of decaying isotropic turbulence.
\end{abstract}

\footnotetext{
${ }^{1}$ This research was supported by the National Aeronautics and Space Administration under NASA Contract No. NAS1-19480 while the first author was in residence at the Institute for Computer Applications in Science and Engineering (ICASE), NASA Langley Research Center, Hampton, VA 23681-0001.
} 


\section{Introduction}

Compressible shear layers are encountered in many practical applications ranging from supersonic ejectors for mixing and entrainment in gas turbines to scramjet combustion. Such flows, important in mixing enhancement and noise reduction, are modified using different nozzle shapes or strategies such as multiple jets, coaxial jets, and countercurrent mixing layers. Compressible mixing layers, for a certain class of initial conditions, have a growth rate substantially lower than their incompressible counterparts. Compressibility effects also contribute to substantial reductions in turbulence intensity levels and the turbulent shear stress anisotropy while increasing levels of anisotropy of the normal stresses. Additional details regarding the effects of compressibility can be found in Bradshaw [1], Lele [2], Spina et al. [3], and Gutmark et al. [4].

Many of these flows are part of a class that have a small bulk dilatation and in which turbulence gradients are more important than mean pressure gradients. Such flows typically have a small turbulent Mach number squared, $M_{t}^{2} \ll 1$, where $M_{t}=\tilde{u} / c, \tilde{u}$ is a turbulent velocity scale and $c$ is the mean speed of sound. This reflects about the weakly compressible nature of the turbulence - the mean flow itself may be highly supersonic. An analytical development of the covariance with the fluctuating dilatation suitable for such a class of flows has been given in Ristorcelli [5]. This note explores the implications of that analysis in the context of the initial conditions for DNS or turbulence model calculations. It is shown that as a consequence of a finite turbulent Mach number there are finite non-zero density, temperature and dilatational fluctuations. Moreover these fluctuations are, to lowest order, specified by the incompressible field. These results are relevant to initial conditions on thermodynamic quantities employed in DNS of compressible turbulence that are started using incompressible fluctuating velocity fields. A potential consequence of the present results is a reduction in the amount of computational effort spent adjusting to transients associated with the relaxation from arbitrary initial conditions. Also, for flows such as decaying isotropic turbulence which are highly dependent on initial conditions, the methodology presented here provides a more natural type of initial conditions than others which have been previously considered.

\section{Analysis}

Ristorcelli [5] has conducted a small Mach number expansion of the compressible NavierStokes equations. Some of those results, as they are relevant to the present subject, are now briefly sketched. The problem of compressible turbulence can be viewed as a singular 
perturbation. The inner problem is related to the acoustic source problem with time and length scales based on the turbulence time and length scales, $\ell / \tilde{u}$ and $\ell$. Here $\tilde{u}$ and $\ell$ are a fluctuating velocity scale and a turbulence integral length scale. The outer problem is the acoustic propagation problem, which in the low $M_{t}^{2}$ limit has time and length scales $\lambda / c=\ell / \tilde{u}$ and $\lambda=\ell / M_{t}$. The inner expansion is relevant to the compressible turbulence modeling problem for compact flows [5].

The velocity field is decomposed according to $u_{i}=v_{i}+\epsilon^{2} w_{i}+\ldots$ thus $d=u_{i, i}=\epsilon^{2} w_{i, i}+\ldots$, where the small parameter is $\epsilon^{2}=\gamma M_{t}^{2}$ where $M_{t}=\tilde{u} / c$. The thermodynamic variables are decomposed according to a perturbation about a mean state, $(P, \bar{\rho}, T)$, thus $p^{*}=P(1+p)$, $\rho^{*}=\bar{\rho}(1+\rho)$ and $T^{*}=T(1+\theta)$. Then ansatzs of the form $p=\epsilon^{2}\left[p_{1}+\epsilon^{2} p_{2}+\ldots\right]$ are used. In the context of turbulence models, the following definition of the fluctuating Mach number is used by $M_{t}^{2}=\frac{2}{3} k / c^{2}$ where $k=1 / 2<u_{j} u_{j}>$. To lowest order

$$
\begin{aligned}
v_{i, t}+v_{p} v_{i, p}+p_{1, i} & =0 \\
v_{i, i} & =0 \\
p_{1, j j} & =-\left(v_{i} v_{j}\right)_{, i j} \\
\rho_{1} & =\frac{1}{\gamma} p_{1} \\
\theta_{1} & =\frac{\gamma-1}{\gamma} p_{1} .
\end{aligned}
$$

In light of the homogeneous shear flow simulations of Blaisdell et al. [6, 7] (the fluctuations were seen to follow a polytropic gas law with coefficient close to the adiabatic value), the adiabatic case is treated. The dimensional form of the linearized adiabatic equation of state is

$$
\frac{\rho^{\prime}}{\bar{\rho}}=\frac{p^{\prime}}{\gamma P}=\frac{1}{\gamma-1} \frac{\theta^{\prime}}{T}
$$

accounting for the last two equations in the zeroth-order expansion above. The zeroth-order problem is the incompressible problem. The fluctuating pressure is not an independent variable but is set by the vortical velocity fluctuations and which also produces the density and temperature fluctuations. On the inner scales the incompressible pressure fluctuations dominate the pressure field. This pressure is called by acousticians the "pseudo-pressure" in contradistinction to the propagating pressure associated with the sound field. The next order expansion for the continuity equation produces a more complex diagnostic relation for the fluctuating dilatation,

$$
-\gamma d=p_{1, t}+v_{k} p_{1, k} .
$$


The relationships between the density, temperature and dilatation as well as the velocity field associated with the dilatation to be derived presently will be called "pseudo-sound" relationships. The dimensional equivalents of the above relationships, using the facts that $\rho_{1}=\rho^{\prime} / \bar{\rho}, \theta_{1}=\theta^{\prime} / T, p_{1}=p^{\prime} / P$ are

$$
\begin{aligned}
\rho^{\prime} & =\frac{1}{c^{2}} p^{\prime} \\
\theta^{\prime} & =\frac{\gamma-1}{\gamma R \bar{\rho}} p^{\prime} \\
-\gamma P d^{\prime} & =p^{\prime}{ }_{t}+v_{k} p^{\prime},{ }_{k}
\end{aligned}
$$

where $p^{\prime}$ is the incompressible pressure fluctuation. In the context of a calculation two different nondimensionalizations are possible. In either case the pressure fluctuations will scale with $\bar{\rho} \tilde{u}^{2}$. For Case 1 the density and temperature fluctuations are scaled with the turbulence fluctuations, $\bar{\rho} \tilde{u}^{2} / c^{2}$ and $\tilde{u}^{2} / c_{p}$ respectively, and one obtains $\rho=p$ and $\theta=p$. For Case 2 the density and temperature fluctuations are scaled with the mean temperature, $T$, and density, $\bar{\rho}$, and one obtains $\rho=M_{t}^{2} p$ and $\theta=(\gamma-1) M_{t}^{2} p$. In either scaling the fluctuating dilatation is obtained from $-d=M_{t}^{2}\left(p_{t}+v_{k} p_{k}\right)$. In both cases it is clear that for finite non-zero turbulent Mach number there are finite non zero fluctuations of density, temperature, dilatation and pressure and there are specific relationships between these quantities.

Obtaining an initial condition for the compressible portion of the velocity field, $w_{i}$, which is consistent with the relation ( 7 ) is somewhat involved. The fluctuating pressure $p_{1}$ needed in (7) is obtained from the Poisson equation (3), and its time derivative can be found by taking the time derivative of (3) and then substituting for the time derivative of the velocity from (1). This give a Poisson equation for $p_{1, t}$,

$$
\left(p_{1, t}\right), j j_{j j}=2\left[\left(v_{k} v_{i, k}+p_{1, i}\right) v_{j}\right], i_{i j}
$$

Solving the two Poisson equations (3) and (11), the dilatation is then found from (7). For homogeneous turbulence the dilatational velocity can be found from the dilatation by working in Fourier wave space. Since the dilatational velocity is irrotational the Fourier coefficients of the velocity are aligned with the wavevector, $\vec{k}$, and they can be found from

$$
\widehat{w}_{j}=-i \frac{k_{j}}{k^{2}} \hat{d}
$$

The dilatational velocity $w_{i}$ is then combined with the solenoidal velocity $v_{i}$ to obtain the full velocity field. 


\section{Simulations and Results}

In order to determine the effectiveness of the new initial condition method, direct numerical simulations of decaying isotropic turbulence are done with three different types of initial conditions. For all three types of initial conditions a solenoidal velocity field is specified with an initial three-dimensional spectrum given by

$$
E(k)=A k^{4} \exp \left(-2 k^{2} / k_{p}^{2}\right)
$$

and random phases for the Fourier coefficients. The constant $A$ determines the amplitude of the velocity fluctuations and is set to obtain a specific turbulent Mach number, $M_{t}$. The peak in the spectrum is given by the wavenumber $k_{p}$ which is set to 12 for all of the simulations presented here. The first method, IC1, specifies zero density and pressure fluctuations. The velocity field is purely solenoidal so that the dilatation is zero. The second method, IC2, has zero density fluctuations, but the fluctuating pressure field is found by solving the Poisson equation (3). The velocity field is again purely solenoidal. The third method, IC3, uses the methodology developed here. The fluctuating pressure field is found by solving the Poisson equation (3). The density fluctuations are related to the pressure through (4). The dilatation is nonzero and is found from ( $\bar{\tau})$. Lastly, the dilatation is used to find the dilatational velocity from (12).

Details about the simulation method can be found in Blaisdell et al. [6]. Simulations were performed with initial turbulent Mach numbers $M_{t}=0.231,0.115$ and 0.058 . The initial turbulent Reynolds number $\operatorname{Re}_{T}=\bar{\rho} q^{4} / \varepsilon \nu=200$ where $q^{2}=\bar{\rho} u_{i} u_{i} / \bar{\rho}$ and $\varepsilon$ is the dissipation rate of turbulent kinetic energy per unit volume.

For the present brief communication one figure, using data from the $M_{t}=0.231$ runs, has been chosen to illustrate the analysis. Figure 1 shows the development of the variance of the dilatation, $\overline{d d}$. It is clear from the figure that there are sizable time boundary layers near the origin for the first two methods as the flow seeks to adjust to inconsistent initial conditions. This is not the case for the pseudo-sound initial conditions. Also interesting to note is the fact that the variance of the dilatation for IC1 is one order of magnitude larger than IC3. In general, at lower $M_{t}$ this difference is more pronounced and at $M_{t}=0.058$ the difference is two orders of magnitude. This excess dilatation is associated with the initial condition and not the dilation due to the evolving compressible velocity field. These results are consistent with the physics of the near field evolution as developed in [5]. 
An intuitive argument can be given to explain the behavior seen in Fig. 1. One might speculate that there is a wave field generated by the inconsistency between the pressure, density, temperature, and dilatational fields whose decay rate is far slower than the turbulence decay. This gives rise to a background acoustic radiation that lasts the course of the simulation. For IC1 the fluctuating pressure field is set to zero. This can be viewed as a combination of the incompressible pressure satisfying (3) plus an acoustic pressure field which exactly cancels the incompressible pressure field. As time evolves the two pressure fields become decorrelated, giving rise to large acoustic pressure and dilatation fluctuations. For IC2 the pressure field is correct; however, there is no dilatation field. Again this can be viewed as a combination of the dilatation found from (7) plus an acoustic dilatation field that exactly cancels this. As the flow evolves away from the initial conditions, the two dilatation fields become decorrelated so that one is left with the correct dilatation plus acoustic fluctuations of dilatation and pressure. The evolution of the pressure variance (not shown here) corroborates this picture.

\section{Conclusions}

The major result of this note is that the initial conditions on the fluctuating thermodynamic variables of density, temperature, pressure, and dilatation should not be arbitrarily specified in the DNS of compressible turbulence. To lowest order these fluctuations, whose nature is connected by the underlying fluctuating vortical turbulence field, are generated by the pressure field associated with the divergence-free portion of the vortical motions. The methodology for generating initial conditions presented here allows the flow to develop more naturally. The significance of the initial conditions may depend on the type of flow considered; however, one is led naturally to speculate about the nature and relevance of simulations that start from arbitrary initial conditions.

A discussion of initial condition issues is given in Blaisdell et al. [7]. In homogeneous shear flow the effects of arbitrary initial conditions are seen to dissipate by $S t=6$, where $S$ is the shear rate. In isotropic turbulence, however, the initial conditions do not fade. Blaisdell et al. [7] has pointed out that the lack of coupling between the wave and vortical fields in the isotropic flow is a probable source of these two different dependencies on initial conditions. (This should also be understood as an additional dependence on the initial conditions other than that already established for incompressible isotropic turbulence.)

There are surely a large number of interesting studies in which one can study the relaxation from diverse arbitrary initial conditions. It is also possible to argue that they have practical 
value. However, the strongest argument, given the current engineering problems and the lack of knowledge regarding the effects of compressibility, can be made for the initial conditions in which the density, temperature, pressure, and dilatational fields are related to the underlying local turbulence field that is, to a large degree, the source of the fluctuations. This seems better than an arbitrary guess at initial conditions that may or may not have physical relevance or meaning and may produce unrealizable results.

\section{References}

[1] Bradshaw, P. (1977). Compressible turbulent shear layers. Ann. Rev. Fluid Mech. 9:33.

[2] Lele, S.K. (1994). Compressibility effects on turbulence. Ann. Rev. Fluid Mech. 26:211.

[3] Spina, E.F., A.J. Smits, S.K. Robinson (1994). The physics of supersonic boundary layers. Ann. Rev. Fluid Mech. 26:287.

[4] Gutmark, E.J., K.C. Schadow, K.H. Yu (1995). Mixing enhancement in supersonic free shear layers. Ann. Rev. Fluid Mech. 27:375.

[5] Ristorcelli, J.R. (1995). A pseudo-sound constitutive relationship and closure for the dilatational covariances in compressible turbulence: an analytical theory. ICASE Report 95-22, submitted J. Fluid Mech.

[6] Blaisdell, G.A., N.N. Mansour, W.C. Reynolds (1991). Compressibility effects on the growth and structure of homogeneous turbulent shear flow. Report TF-50, Department of Mechanical Engineering, Stanford University, Stanford, CA.

[7] Blaisdell, G.A., N.N. Mansour, W.C. Reynolds (1993). Compressibility effects on the growth and structure of homogeneous turbulent shear flow. J. Fluid Mech. 256:443. 


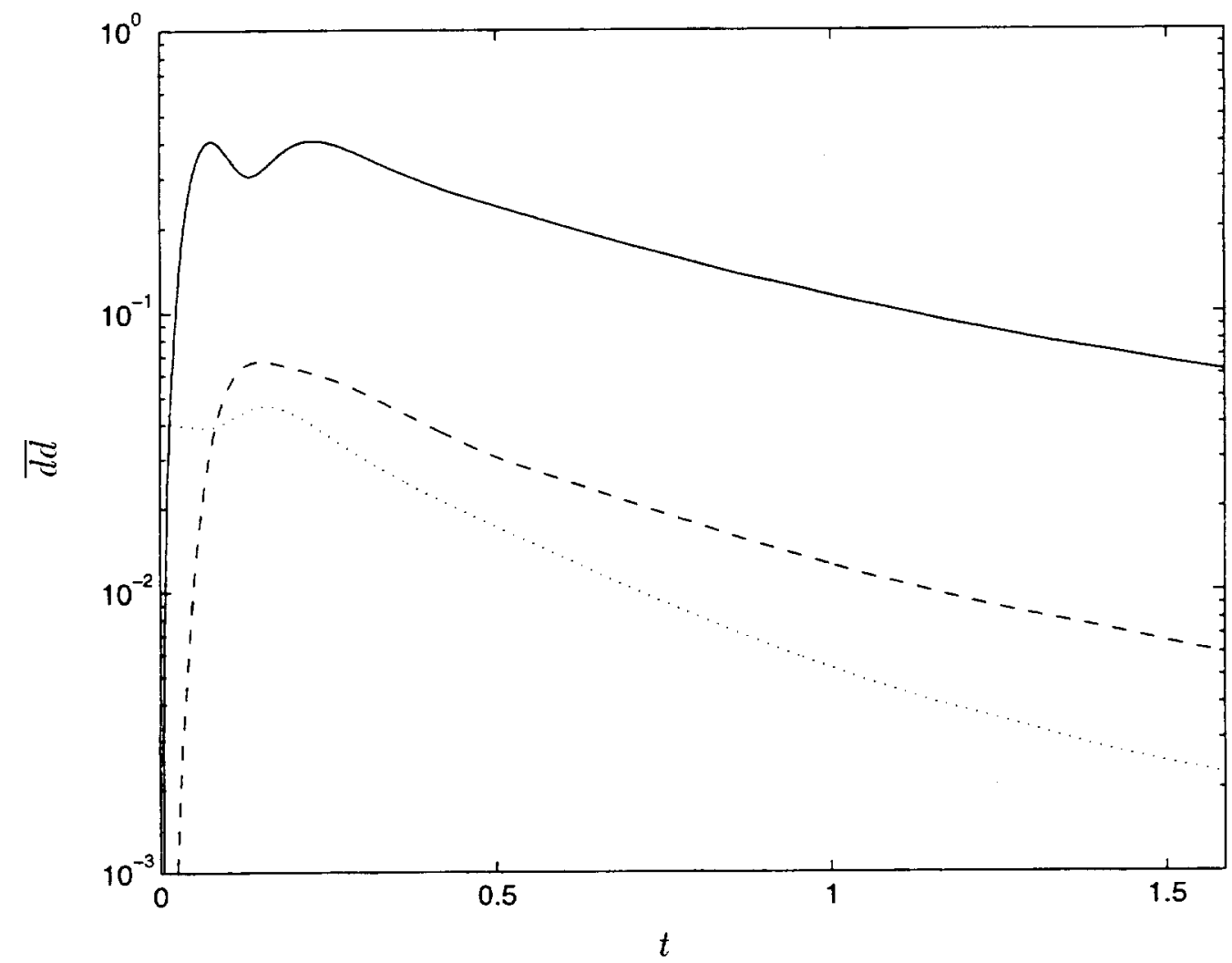

Figure 1: Time history of the dilatation variance for decaying isotropic turbulence using initial conditions IC1 ( - ), IC2 ( --- ), and IC3 ( ….... ). 
. 

Public reporting burden for this collection of information is estimated to average $I$ hour per response, including the time for reviewing instructions, searching existing data sources. gathering and maintaining the data needed, and completing and reviewing the collection of information. Send comments regarding this burden estimate or any other aspect of this collection of information. including suggestions for reducing this burden, to Washington Headquarters Services. Direciorate for information Operations and Reports, 1215 Jefferson Davis Highway, Suite 1204, Artington. VA 22202-4302, and to the Office of Management and Budget, Paperwork Reduction Project (0704-0188). Washington. DC 20503

\begin{tabular}{l|l|l} 
1. AGENCY USE ONLY(Leave blank) & $\begin{array}{c}\text { 2. REPORT DATE } \\
\text { July } 1996\end{array}$ & $\begin{array}{l}\text { 3. REPORT TYPE AND DATES COVERED } \\
\text { Contractor Report }\end{array}$
\end{tabular}

4. TITLE AND SUBTITLE

THE DNS OF COMPRESSIBLE TURBULENCE

\section{FUNDING NUMBERS}

C NAS1-19480

WU 505-90-52-01

6. AUTHOR(S)

J. R. Ristorcelli

G. A. Blaisdell

7. PERFORMING ORGANIZATION NAME(S) AND ADDRESS(ES)

Institute for Computer Applications in Science and Engineering

Mail Stop 132C, NASA Langley Research Center

Hampton, VA 23681-0001

8. PERFORMING ORGANIZATION REPORT NUMBER

ICASE Report No. $96-49$

9. SPONSORING/MONITORING AGENCY NAME(S) AND ADDRESS(ES)

National Aeronautics and Space Administration

Langley Research Center

Hampton, VA 23681-0001

10. SPONSORING/MONITORING AGENCY REPORT NUMBER

NASA CR-201589

ICASE Report No. $96-49$

\section{SUPPLEMENTARY NOTES}

Langley Technical Monitor: Dennis M. Bushnell

Final Report

To be submitted to Physics of Fluids A.

12a. DISTRIBUTION/AVAILABILITY STATEMENT

12b. DISTRIBUTION CODE

Unclassified-Unlimited

Subject Category 34

13. ABSTRACT (Maximum 200 words)

Relationships between diverse thermodynamic quantities appropriate to weakly compressible turbulence are derived. It is shown that for turbulence of a finite turbulent Mach number there is a finite element of compressibility. A methodology for generating initial conditions for the fluctuating pressure, density and dilatational velocity is given which is consistent with finite $\mathrm{Mach}$ number effects. Use of these initial conditions gives rise to a smooth development of the flow, in contrast to cases in which these fields are specified arbitrarily or set to zero. Comparisons of the effect of different types of initial conditions are made using direct numerical simulation of decaying isotropic turbulence

14. SUBJECT TERMS

Compressible Turbulence; DNS; Initial Conditions

15. NUMBER OF PAGES

9

16. PRICE CODE

$\mathrm{AO} 2$

17. SECURITY CLASSIFICATION OF REPORT

Unclassified

18. SECURITY CLASSIFICATION OF THIS PAGE

Unclassified
19. SECURITY CLASSIFICATION OF ABSTRACT
20. LIMITATION OF ABSTRACT

NSN 7540-01-280-5500 